\title{
To understand and be understood: facilitating interdisciplinary learning through the promotion of communicative competence
}

\author{
Stephen Taylor \\ School of Geography, Queen Mary University of London, Mile End Road, London, E1 4NS. \\ United Kingdom. \\ Email: stephen.taylor@,qmul.ac.uk; Tel: +44 (0)2078822748
}

\begin{abstract}
Whilst interdisciplinarity has become a central concern of research and learning in geography, few from the discipline have considered the practical facilitation of interdisciplinarity in the classroom. Module convenors, I argue, must pay greater attention to how learners engage and negotiate with peers and perspectives from other disciplines. In this paper, I focus on my own efforts in designing and teaching a second year undergraduate course on health, biomedicine, and society, to illuminate the opportunities and challenges facing teaching staff who seek to provide opportunities for interdisciplinary learning in the classroom. Drawing upon theories of communication, I demonstrate the significance of developing "communicative competence" as a pathway to successful interdisciplinary learning. Unless learners from one discipline can understand, and be understood by, peers from other disciplines, the accomplishment of interdisciplinary learning is undermined. Interdisciplinary modules should include learning outcomes that facilitate student development in this area. Approaching interdisciplinary learning through the lens of communicative competence casts critical attention upon the central abilities and cultural sensitivities that are the hallmarks of interdisciplinary collaboration from negotiating meaning to critical disciplinary awareness - and highlights the lessons that interdisciplinarity poses for disciplines, such as geography, tasked with preparing students for interdisciplinary learning.
\end{abstract}

Key words: interdisciplinary learning; interdisciplinarity; interdisciplinary communication; classroom communication; teaching and learning

\section{Acknowledgements}

First, I would like to thank the students of "Health, Biomedicine, and Society" whose engagement, comments and feedback have profoundly shaped my own teaching and scholarship in recent years. I also acknowledge a significant debt to David Andrew, David Beckingham, Tim Brown, Fran Darlington-Pollock, Al James, Francesca Moore, David Nally, Simon Reid-Henry, Caroline Walker, and Matthew Williamson for encouraging me - through both their advice and example - to cast a critical eye over my own teaching practice. 


\section{Introduction}

Research and learning that crosses traditional disciplinary domains is of growing significance within higher education (Davies, Devlin \& Tight, 2010; Lyall, Meagher, Bandola \& Kettle, 2015). While acknowledging that the idea of "a discipline" disguises wide variations in practice, authors associate disciplines with distinct cultures, methods, and epistemologies (Schoenberger, 2001; Barry, Born \& Weszkalyns, 2008). Disciplinary socialisation configures approaches to research and learning, and this can, in turn, lead to frustration and miscommunication when those from different disciplinary backgrounds attempt to collaborate. The extensive literature on the politics of interdisciplinary research testifies to the difficulties of collaborating across disciplinary divides (Bracken \& Oughton, 2006). Proponents of interdisciplinary learning have, likewise, reflected on the opportunities and challenges of facilitating learning across disciplinary boundaries (Elder, 1999; Foster, 1999; Pharo \& Bridle, 2012). Prominent arguments in support of interdisciplinary learning include: the critical engagement that students develop with the limitations of learning based within one discipline; the suggestion that interdisciplinary collaboration is a vital skill to prepare graduates for new, team-based forms of employment; and, the growing realisation that tackling contemporary planetary challenges (such as climate change, urban growth, and global health) will need alternative, integrated approaches (Castree, 2016). And yet just as interdisciplinary research was, and often still is, hampered by the slow adaptation of supporting institutional structures, interdisciplinary learning has been held back by a continued reliance on "the discipline" as the recruiter of students and the provider of taught programmes. ${ }^{1}$ Individual teaching staff are, thus, expected to provide both rigorous programmes of study within their discipline while also developing interdisciplinary learning opportunities.

Advocates of interdisciplinarity have long considered geography to be a key disciplinary ally (Lau \& Pasquini, 2008; Simon \& Graybill, 2010). Geography, according to Skole (2004, p. 742 ), is "the great interdisciplinary melting pot" and provides a base for interdisciplinary "immigrants" from other, less-transgressive disciplines. Likewise, Baerwald (2010, p. 497) suggests that "[i]f disciplines are tribes with distinctive cultures, geographers are a breed inclined to wandering, exploring and working together with others." While geographers have had much to say about the significance of their "melting pot" discipline to interdisciplinary

\footnotetext{
${ }^{1}$ While interdisciplinary undergraduate degree programmes remain few and far between (in the English context at least), the growth and success of interdisciplinary postgraduate programmes is encouraging (see Donovan, Sidaway \& Stewart, 2011).
} 
research, they have said less about the discipline's contribution to nascent interdisciplinary learning agendas. ${ }^{2}$ Indeed, while a growing number of geography departments report institutional expectations to contribute expertise to interdisciplinary taught programmes, teaching staff report frustrations about the poor coordination of content and quality assurance mechanisms between modules in such programmes (Bradbeer, 1999). Faced with these expectations and challenges, it is important that geographers consider how best to enable interdisciplinary learning through their taught programme offerings.

Before considering how geographers might best respond to such agendas, it is important to define the term "interdisciplinary learning." I take interdisciplinary learning to involve collaborations between learners from differing disciplinary specialisms who, through the pooling of their existing disciplinary knowledge, work together to examine and address complex, real world issues (Strober, 2006). Specialist modules in which associate learners (those from different university departments) are welcome to take part rarely fall within this understanding of the term. Such modules are better understood as contributions to "multidisciplinary" learning, in which additional specialist knowledge supplements existing disciplinary knowledge, with little concerted effort made at connecting distinct knowledge domains. Interdisciplinary learning, in contrast, involves the bridging and synthesis of disciplinary knowledge domains to examine and solve complex problems. At institutional level, the Universities of Melbourne and Aberdeen have trialled interdisciplinary models with varying degrees of success (James \& McPhee, 2012; Onsman \& Newton, 2015). ${ }^{3}$ While these institution-wide models have garnered much critical attention, the practical operation of interdisciplinarity in the classroom itself remains underexplored. Once students from different disciplines enrol on a course, for instance, how does the module leader go about facilitating interdisciplinary skills? I suggest that the key learning outcomes of interdisciplinary modules ought to be the ability to understand different disciplinary perspectives and to likewise be understood by peers; by implication, a central concern of interdisciplinary modules must be the facilitation of effective communication. My intention here is to better understand if interdisciplinary communication is different to other forms of collaborative learning and, if it is, how teaching staff might better structure interdisciplinary learning to foster such

\footnotetext{
${ }^{2}$ As ever, there are exceptions to this trend. Jones and Merritt (1999), Schmelzkopf (2002), and Herrick (2010) document their own efforts, as geographers, to promote interdisciplinary learning in the classroom.

${ }^{3}$ The development of these flagship interdisciplinary models, one could suggest, is as much about marketing the distinctiveness of the institution in the competitive neoliberal world of higher education as it is about incubating interdisciplinary best practice.
} 
communication. But what are the characteristics of interdisciplinary communication, and how can module leaders provide opportunities to develop these in practice?

To examine communication in the interdisciplinary classroom, I focus on my own experience of developing and teaching a second-year undergraduate module on "Health, Biomedicine, and Society" at Queen Mary University of London (QMUL). The module is interdisciplinary in scope and draws many students from a broad selection of disciplinary backgrounds, with the largest cohort drawn from the School of Geography. Drawing upon communication theory, I begin by proposing that "communicative competence" must be a key learning outcome for modules that seek to facilitate learning across distinct disciplinary cultures. Where critics have decried extant accounts of interdisciplinary communication for drawing caricatures of disciplinary cultures, I seek instead to illuminate actual student experiences of interdisciplinary learning. Drawing upon interviews with 30 students from the module, I then examine in detail student understanding, engagement, and learning regarding seven specially-formulated learning objectives that each focused on developing a different aspect of interdisciplinary communication. My aim in analysing student engagement with these interdisciplinary learning outcomes is threefold: first, to explore how best to develop communication as a core interdisciplinary ability; second, to scrutinise how communication in an interdisciplinary classroom facilitates the critical evaluation of disciplinary and interdisciplinary cultures; and, finally, to provide a sound empirical study of the possibilities and pitfalls of interdisciplinary learning. In the concluding section of this paper, I close by expanding upon the specific implications of communication-focused interdisciplinary learning for the discipline of geography, particularly as concerns the discipline's responsibility for preparing undergraduate students for interdisciplinary learning.

\section{The importance of communication in the interdisciplinary classroom}

Scott (2002, p. 70) describes the English higher education system as a domain in which "the subject is still king." This scarcity of opportunities to engage in interdisciplinary learning is not unique to the English context (Pharo et al., 2012). It seems that elsewhere, too, students engage with interdisciplinary learning in a haphazard manner - more akin to the multidisciplinary approach I defined earlier - selecting individual optional modules from different schools and receiving little support in drawing connections between these modules and their own programme of study. Such systems assume learning to resemble the process of osmosis, in which core disciplinary knowledge and perspectives are diffused over the course 
of a module to those in the class from outside the discipline. In contrast, I take interdisciplinary learning to be a systematic attempt to emulate the operation of a multi-professional team in which individuals communicate insights and experiences learned from their own specialities before integrating this knowledge to negotiate a suitable solution to a complex problem. As such, I propose that interdisciplinary learning is best introduced to students already partly socialised into the methods, cultures, and epistemologies of their discipline (undergraduates in the penultimate or final years of study) and who have sufficient disciplinary perspectives to communicate to others in the negotiation of new approaches to complex, shared problems.

From similar starting premises, Becher and Trowler (2001) conceptualise how students from different disciplines come to both understand and transcend their socialisation into their respective disciplines. Disciplinary cultures, they propose, are "sets of taken for granted values, attitudes and ways of behaving, which are articulated through and reinforced by recurrent practices" (Becher \& Trowler 2001, p. 23). Communication is key in promoting knowledge exchange across otherwise distinct cultures and "bind[s] together the sociological and epistemological, giving shape and substance to the links between knowledge forms and knowledge communities" (Becher \& Trowler 2001, p. 14). As a mode of articulating difference, then, communication is key in bridging disciplinary cultures; any attempt at promoting interdisciplinary learning must seek to develop communication skills such that learners already socialised into academic disciplines might be better able to establish common ground with peers from outside their discipline. In the interdisciplinary classroom, however, the outcome of learning cannot merely be the ability to communicate with others; rather module leaders must seek to develop the competence of learners to communicate and problem-solve effectively. Besides acquiring knowledge on the subject or problem at hand, students working in an interdisciplinary classroom might have to develop such skills as: exploring what each constituent discipline can best contribute to the project; considering (and discounting) different sources and forms of evidence; synthesising data and concepts into a singular analytical framework that is agreeable to all parties; and, presenting the outcomes of this learning experience in a manner that is accessible to an interdisciplinary audience. Module leaders should, I believe, include learning outcomes crafted to accomplish aims such as these within module design to give due prominence to the centrality of communicative competence as a key outcome of interdisciplinary learning. 
Hymes (1972) developed the concept of "communicative competence" as a rejoinder to Chomsky's (1965) work on "grammatical competence," or the ability of an individual to recognise the distinct grammatical structures of language and then reproduce them through effective communication. In developing Chomsky's focus on competence in syntax, Hymes' ethnographic research on sociolinguistics identified the key role of both form and function that is, the use of correct and appropriate syntax - in communication between individuals. Communication scholars have since developed this line of reasoning to theorise communication between different "cultures," be that those defined by gender, ethnicity, or nationality. In the literature on education, a focus on communicative competence has now expanded beyond studies of foreign language education to include work concentrating on comprehension and cooperation across the distinct disciplinary cultures of higher education (Canale, 1983; Alptekin, 2002). Enduring differences, of course, remain in the form and function of communication within different disciplinary cultures and teaching staff must embrace, rather than shun, these differences in the interdisciplinary classroom.

In the rest of this paper, and motivated by Hymes' work, I draw upon two models that outline how learners can interrogate, negotiate, and curtail disciplinary differences in communication as they work together in interdisciplinary teams. Byram (1997) draws on intercultural communication theory to conceptualise communicative competence in learning. For Byram, the development of communicative competence necessitates learning across five domains (attitudes, knowledge, skills of interpreting and relating, skills of discovery and interaction, and critical cultural awareness). Woods (2007) develops this model further by fragmenting the "knowledge" domain into a further three elements (conceptual competence, competence in negotiating meaning, and competence in interdisciplinary text production) for inclusion in interdisciplinary curriculum design. These models provide a conceptual framework for approaching learning and communication across disciplinary boundaries. With some adjustment, as I will outline, module leaders can frame these domains of communicative competence as formal learning outcomes for interdisciplinary modules that seek to enable the development of the knowledge, skills, and attitudes necessary for effective communication across disciplines. 


\section{Module context, learning outcomes and methods}

"Health, Biomedicine, and Society" is a two semester, second year undergraduate module that was first taught in 2013-14. The module focuses on the social, political, and ethical debates surrounding health, health care, and biomedical knowledge production globally. While the School of Geography hosts administrative and quality assurance oversight, this module is open to students from across the university and they do not need any specialist health-related knowledge or prerequisites. This willingness to welcome students from outside the School reflects an individual commitment to interdisciplinarity on the part of the convenor, but also connects to new institutional initiatives such as the launch of a multidisciplinary BSc Global Health (based in the School of Medicine) on which the module is an optional choice. The 20152016 version of the module involved a total of 73 students from seven disciplines (geography, politics, law, medicine, English, engineering, and business management). Geography undergraduates represented the largest cohort and accounted for 40 percent of student enrolments.

The taught component of the module consists of 16 two-hour interactive lecture classes and six one-hour small-group seminars. In the lecture classes, students contribute to a selection of weekly formative assessments - including short presentations, role-plays, and policy critiques - in a changing set of self-selected monodisciplinary and multidisciplinary small groups. For seminar classes, seven to eight students are assigned to a multidisciplinary group by the convenor in an attempt, where possible, to have at least one representative of each discipline in each of the small groups. The seminars focus on discussion of pre-circulated readings and collaborative exercises involving, for instance, unpicking the entangled geographies of generic paracetamol production using Cook et al.'s (2004) "follow the thing” approach. The module assessment consists of two 2,000-word coursework reports on contemporary medical or ethical debates (both $25 \%$ of the module mark) and an unseen examination (50\%). The coursework components involve students writing individual research reports on selected topics related to lectures (including bioprospecting, the sale of human organs, and the globalisation of clinical trials), and then reflecting on how formative discussions with students from disciplines other than their own informed the direction of this research.

To gauge the development of communicative competence among the students in this interdisciplinary classroom, I conducted individual semi-structured interviews with 30 students at the beginning, middle, and end of the module. I chose a longitudinal qualitative approach to 
capture the dynamics and diversity of student experience on the module; this approach complemented the routine institutional capture of quantitative metrics of learning gain (via grades and student self-assessment surveys) at the beginning and end of modules. I selected at least two students from each of the seven disciplines represented, with the rest of the students in the sample selected according to the proportion of their discipline in the class. ${ }^{4}$ I presented students with revised learning outcomes of the module, introduced in 2015-16 to place a greater emphasis on developing interdisciplinary communication, each of which I had drafted to correspond to one of Byram's (1997) and Woods' (2007) domains of communicative competence (see Table 1). I then used this list as a discussion prompt to encourage the students to reflect on their previous learning experiences and perceived personal competence in each of these seven domains. In the pre-module interviews, this discussion allowed me to gauge student expectations (and anxieties) about an interdisciplinary module and benchmark familiarity with the module's learning outcomes. In the interviews conducted during and after the module, I used the same discussion prompts to capture changes to students' perceived competence in relation to these outcomes. Following the completion of the module, I compared this qualitative data to quantitative measures captured by QMUL to measure whether students evidenced competence in each of the learning outcomes through the combined elements of summative assessment. While my focus in what follows is on student experience revealed through the qualitative research, I draw connections to the institutional quantitative measures in the final section of the paper.

[Table 1 near here]

\section{Interdisciplinary abilities}

The first three learning outcomes are informed by Woods' (2007) main interdisciplinary abilities that emerge from communicative competence: "conceptual competence," "competence in negotiating meaning," and "competence in interdisciplinary text production." In what follows, I give examples of how I sought to facilitate these abilities in the classroom and examine comments and feedback from students on their engagement with each of these learning outcomes and interdisciplinary abilities.

\footnotetext{
${ }^{4}$ The QMUL research ethics committee approved this research. Students were enthusiastic about the research and all those approached agreed to take part. All participants signed an informed consent form that made clear that their (non-)participation would not influence their final grade. Interviews were scheduled in the first week of the first semester and the first and final weeks of the second semester. Interviews were recorded, transcribed, and then thematically coded. All 30 students completed the schedule of three interviews.
} 


\section{Conceptual competence}

This domain represents the ability to distinguish, explain, and put in place pertinent methods and concepts from different disciplines to solve a problem. For Woods (2007, p. 859), these methods represent the articulation of familiar disciplinary concepts in pursuit of addressing a problem. In developing my own learning outcomes, however, I chose to develop this domain further to acknowledge the importance of learning how to apply knowledge of familiar and unfamiliar concepts, from a variety of disciplines, to address complex situations. The ability to understand, integrate, and develop familiar and unfamiliar concepts is a domain of communicative competence that is just as essential to interdisciplinary problem-solving as depth of knowledge in a particular area. Solving complex problems requires a mastery of familiar conceptual approaches but it also requires a creative approach to integrating and developing unfamiliar ones.

Most students on the module were initially uneasy about their personal competence in explaining concepts from their own disciplines and were, likewise, unsure about their ability to understand new concepts from outside their discipline. One frequently aired anxiety among the social science and humanities students concerned explaining theoretical concepts to peers well-versed in positivist and quantitative approaches. "If I'm honest," one geographer confessed, "I understand a lot of the social theories mentioned in lectures - Foucault, Marx, et cetera - but explaining that to someone, to who it is entirely new, I feel is a little bit beyond my comfort zone." Interestingly, most of the students from the natural and medical sciences were equally unsure about how much their own suite of approaches would be embraced by peers from non-scientific backgrounds. "I guess we are going to be salespeople for a set of methods and approaches that some of the class haven't considered since the end of secondary school," one medical student concluded.

During the module, multidisciplinary groups of students were set short tasks to complete in class. One such task involved using a set of academic and journalistic accounts to consider if a free market in human organs would end some of the social and ethical issues that arise from the current black-market trade. Six representatives were then nominated to take part in a competitive Oxford-style debate framed around the motion "This house believes that human organs should be sold freely" (with three speakers "for" and three speakers "against"); class members voted both before and after the debate, with the winners considered to be the team 
that convinced the most members of the audience to change their original vote. Many students discussed this exercise as a highlight of the module. "We had one of the business students talk through what a market for organs would look like," one of the engineering students revealed, "and we had lawyers pointing out places where technical or regulatory issues would arise." Several interviewees referred to other sessions where they acquired knowledge of unfamiliar concepts - such as creative thinking techniques - from group members on different degree programmes. For others, as one law student noted, familiar methods, such as problem-based learning, used in unfamiliar disciplines produced an unexpected sense of shared understanding: "One of the medical students suggested we approach the problem by role-playing cases with each of us representing different stakeholder perspectives and I actually felt immediately at home because of the parallels with the legal case method."

While most students interviewed reported a growing familiarity with tools and concepts from other disciplines, interviews conducted at the end of the module revealed certain limits to peer communication in this setting. Competence with quantitative approaches remained an area of confusion and misunderstanding for many in the class - " $[\mathrm{t}]$ he engineering students lost us in some of the technical discussion on bioengineering," noted one geographer - although all the students interviewed from the STEM disciplines reported increased confidence in the use of qualitative approaches because of learning and peer discussion across the module. This finding, echoing broader concerns about quantitative literacy in university graduates, suggests that module leaders ought to prioritise further training aimed at raising confidence with both qualitative and quantitative methods to prepare students to make the most of interdisciplinary learning opportunities.

\section{Competence in negotiating meaning}

Woods (2007, pp. 859-860) makes the case that interdisciplinary communicative competence also enables a student to "recognise when specialist terms may require explanation." The second learning outcome for the module built upon this domain by emphasising that interactions between learners from different disciplines are unlikely to be successful unless adequate time is taken to develop mutually agreed upon definitions of key terms. In a case that will be familiar to many geographers, James (2006, p. 302) suggests that the term "culture" is illustrative of the way the meaning of taken-for-granted terms varies across different knowledge domains, concluding that there are many conflicting definitions of this "fuzzy" term in circulation. Learning focused on negotiating the meaning of key terms is essential for 
students to develop competence in communicating disciplinary knowledge to those from other disciplines and also going beyond this to establishing agreed upon understandings of common terms.

The students interviewed spoke at length on the complexity of these negotiations. Few of the students had considered the question of discipline-specific definitions of key terms before their participation in the module. According to one of the engineering students, "health is the absence of disease." And yet one of the geographers noted, "we have to consider the broader issue of well-being and whether we have things like rights to health." These two illustrative comments from the pre-module interviews were a timely reminder of the importance of facilitating discussion around the meaning of significant, yet contested, terms. In the first class of the module, I gave each student the task of defining what they understood "health" to be. This produced a wide variety of different responses, but discussion turned to disciplinary specifics when one student proposed to the class that a representative of each discipline support their definition with an illustration taken from one of their previous modules. One student explained the classroom dynamic during this discussion:

"It was the discussion around definitions that got me excited about this interdisciplinary class. You had a medical student complaining that the geographers were using the word 'inequality' when the real issue was 'inequity.' We used that to time to reflect on why our disciplines chose to focus on particular definitions and not others. It was eyeopening."

Indeed, this impromptu discussion set the tone for later classroom exercises and group work, with the definition of key terms proving to be the source of frequent extended dialogue. "The term 'market' meant something different to each member of our group," noted an English student, "[t]he medical student was scared about privatisation of health care, yet the business student saw it as a source of new efficiencies. Conversation got heated!"

According to the end of module interviews, students considered the negotiation of shared understandings to be a key success of the module. "You realise how 'tight' you have to be in communicating with others," said a geographer, "always considering how best to make a point to solve the problem, and that's a real learning accomplishment." The success of these efforts became evident in assessed work, with both myself and a colleague reviewing the work noting 
the lengths to which students had gone in problematizing understandings of key concepts. The brief student reflections presented above reinforce the significance of giving space and time in the interdisciplinary classroom for peer-to-peer discussion of key terms and definitions.

\section{Competence in interdisciplinary text production}

This third domain promotes learning geared toward the ability to perceive difference in the form and content of communication between disciplines and to then mediate these differences to produce verbal and written outputs that are accessible to an interdisciplinary audience. There are different disciplinary expectations about the form and content of formal and informal modes of communication. In drafting the third learning outcome of this module, I emphasised that students would accomplish learning in this domain of communication through the negotiation of these distinct disciplinary approaches to communication across alternative modes of presentation (including research reports and oral presentations).

Few of the students interviewed at the beginning of the class had experience of collaborating in the production of written materials, but few envisaged any problems with such an exercise. Disciplinary differences soon became plain both to the students and myself with the first submission of draft individual coursework essays. Students from the natural sciences utilised a structured written style - replete with cascading sub-headings - that enabled the reduction of ambiguity, while the social science and humanities students often prioritised style and complexity over brevity and clarity. Students then provided formative feedback on the draft work of a student from another discipline. This provided a significant opportunity for students to learn about the negotiations necessary in producing a text that would communicate their findings to an interdisciplinary audience. As one law student commented:

"It was quite a shock to see the different writing styles all laid out on the table. Some had footnotes, others used diagrams and maps. I realised at that moment that the best text would be an amalgam of these different perspectives, but actually figuring out what to take away from each disciplinary approach took a lot of negotiation and discussion."

Following the discussion of drafts, small groups spent time discussing differences in content and style between their written work. As I wandered the classroom, it was encouraging to hear students asking peers for advice on how to portray the latest developments in human cloning to those without a significant understanding of basic biology and how an English student might 
adapt their written report to pre-emptively answer questions raised by a reader trained within the scientific tradition.

"You make assumptions about your audience when you write," noted a geographer, "but when you meet that audience - people from different disciplines - it means that you have to learn how best to communicate your ideas." As this student summarised, negotiations involving students from different disciplines need a higher order of communicative competence than would be required when working with those from the same or allied fields. Of concern, though, was a more general reluctance to engage in group assessment, particularly oral presentations, where students raised concerns about different levels of individual contribution. Interdisciplinary text production, as a collaborative exercise, requires group work and it is essential that module leaders socialise students at an early stage into the centrality of collegiality to the successful completion of group tasks.

\section{Interdisciplinary culture}

The second set of four learning outcomes relate to the development of student learning in domains related to "interdisciplinary culture." I understand this to mean a developing appreciation of distinct disciplinary norms and a growing awareness of the unique collaborative opportunities that exist across and between these disciplinary domains. Byram's (1997) elements of communicative competence provide a key starting point here, and I relate the learning outcomes for this module to his domains of "interpreting and relating," "attitudes," "discovery and interaction," and "critical disciplinary awareness."

\section{Interpreting and relating}

The fourth learning outcome of the module sought to develop among the class the ability to make sense of a text or presentation from another discipline, to interpret it in light of the course content, and finally compare it to a text or presentation from the home discipline. The perception that texts or presentations from other disciplines are "abstract" or "technical" can frustrate communication between individuals unfamiliar with the outputs of research in another discipline. Learning in this area should begin with the identification of how socialisation into a discipline comes to shape the form and content of outputs. Learners manifest communicative competence in this area when they can identify the disciplinary inferences contained within key documents and relate these to similar inferences in the outputs of their own discipline. 
Students on the module initially reported a familiarity with documents taken from other disciplines. This was particularly the case for students in the humanities and social sciences where items on reading lists were frequently drawn from outside of the home discipline. "In my English classes," one student noted, "we're often reading books from politics on revolutionary identities or from geography on urban issues." Yet most students who had engaged with these texts reported not understanding certain aspects of the texts or questioned the relevance of certain lines of argument contained within them.

In preparation for one class, I instructed students to find a text from within their own discipline about neuroscience and to then find a paper from outside their discipline on the same topic. Students were to read both texts and compare the content and form of the papers. Most respondents expressed surprise at the scope and breadth of arguments outside their discipline. Often this confounded pre-conceptions about other disciplines and, in several cases, gave rise to sophisticated critiques of disciplinary texts in light of arguments developed outside the discipline. One medical student noted:

"I compared some work on neural imaging from medicine with some research from sociology on governing behaviours through the brain. I'm not sure that kind of social theory work would get published in medicine, but it had a lot of traction and made me think about how much the research scientists are being asked to do is actually implicated in another series of murky agendas."

Other students, however, found the exercise to be less revealing and this stemmed from a limited engagement with the text from their home discipline rather than the one from an unfamiliar discipline. "The papers from our discipline were just normal, you take it for granted, and it was quite hard to see how you could say anything new about them," said one politics student. Such statements are a reminder of the importance of preparing critical thinking skills through disciplinary training and encouraging students, before they engage with texts from outside the discipline, of what makes their own discipline's outputs so distinct.

\section{Attitudes}

Inquisitiveness about other disciplinary cultures is of importance in the interdisciplinary classroom, as is a receptiveness to having new understandings shape one's own attitudes. The development of communicative competence in this domain is associated with the readiness of 
learners to engage and interact with peers to compare the cultural practices of other disciplines. Byram (1997, p. 50) argues that this is not "seeking out the exotic or [s]eeking to profit from others," but rather the "willingness to seek out or take opportunities to engage with others in a relationship of equality." In including this domain of Byram's model into the fifth learning outcome for this module, I sought to move beyond an ends-focused approach to interdisciplinary interaction (one focused on solving a problem or learning new approaches) to emphasise the significant role that discovering and understanding disciplinary attitudes plays in facilitating interpersonal interactions.

Interviews with students revealed a broad spectrum of attitudes towards peers from other disciplines. Attitudes towards other students ranged from the stereotypical ("the only thing the politics students are any good for is arguing a lot") to the nuanced ("law students don't just study legal cases in the same way that we [English students] don't just read books all day"). The latter understandings were more common when students reported a positive engagement with group members. "It changed my perception of a lot of the social science disciplines," a medical student commented, "everyone in my group was alert to the potential of science and not as critical as I thought they would be." Unfortunately, negative attitudes towards other disciplines were associated with the perceived weakness or lack of contribution of certain students, rather than because of their disciplinary background per se.

One module provides only a limited opportunity for destabilising prevalent attitudes between disciplines; the fact that many senior researchers share caricatured views of other disciplines shows how tenacious these lines of thinking can be (Callard \& Fitzgerald, 2015). While student feedback suggests that interdisciplinary modules such as this do offer students the chance to engage with other disciplinary cultures in a relationship of equality, it is pertinent to consider how informal interdisciplinary interactions can be better facilitated before such collaboration is assessed (through, for example, non-credit bearing interdisciplinary modules at earlier stages of individual degree programmes).

\section{Discovery and interaction}

The penultimate learning outcome of the module sought to develop communicative competence to equip the learner with the skills to develop a basic understanding of another discipline's culture and processes in an expeditious manner. Interdisciplinary interaction has at its heart the ability to obtain new knowledge of another discipline's cultures and practices 
through interaction with peers from that discipline. This may also involve diagnosing the differences between disciplinary approaches to a problem, and then working with others to enable an effective interaction between advocates of these approaches.

While most students had interacted with peers from other disciplines in social settings, few reported interacting with such students in formal learning situations. Student experiences of taking modules outside of their discipline were mixed. One politics student, for instance, had previously taken a history module and felt isolated because of his unfamiliarity with historical methods and forms of inquiry. "I sat on my own," he said, "because everyone else had their groups already, and that meant I had no-one to bounce ideas off or ask even basic questions about the module." Several sessions in the module provided opportunities for students to develop in this area. I gave students a selection of papers from different disciplines and encouraged them to compare the abstract of these papers to identify how disciplines communicate knowledge. They were then encouraged to try and re-write the abstract in their own disciplinary style, before sharing their efforts with other group members. Many students found this a particularly tricky task to complete, with many struggling to balance the demands of brevity and accuracy characteristic of different disciplines. Yet the high degree of interaction involved in this exercise led others to develop new lines of communication. "In these short exercises," one engineering student said, "you mix with different subjects, get different perspectives, and get insights from students on why they do things a particular way." While it is difficult for a module leader to facilitate the "discovery" of these skills and social connections in the classroom setting, this is something that home disciplines can seek to nurture earlier in degree programmes by encouraging students, for instance, to engage in peer-assisted study.

\section{Critical disciplinary awareness}

The final learning outcome supported developing communicative competence to assess the viewpoints, methods, and outputs of the home discipline and other disciplines. Skills developed in an interdisciplinary module such as this prepare learners to interrogate the implicit and explicit cultures, values, and practices communicated through socialisation into a discipline; they also equip graduates with the critical thinking skills valued by employers.

To illustrate student engagement with this learning outcome, I include here a lengthy quote from a geography undergraduate speaking about the benefits of having peers from other disciplines (particularly those from medicine) point out the limitations of geographical 
perspectives. In a discussion exercise on the issue of public health communication in the Global South, peers challenged this geography student on the seeming simplicity of calls for greater community involvement in health promotion:

"In geography, we pretty much take for granted that we need to consider the role of non-state actors when we are discussing policies. So, it sounded pretty safe to suggest that bringing the community in to help determine public health messages was a good idea. But there were these other perspectives that we don't talk about in geography. I don't know why. Who is the community? What do you do when people lack education? What about paternalism around health in the Global South? It turns out that professional thinking around these issues is much more complicated than we usually get a sense of in geography and the social sciences."

Statements such as this suggest a growing critical awareness of the home discipline, but also an awareness of its fluctuating position within the wider university. "There is no longer that sense that such and such a subject belongs to geography," one geographer noted, "and that means there are lots of opportunities for us to learn new things outside of that narrow disciplinary specialism." While critical disciplinary awareness might be an advanced skill for a second year undergraduate, such insights position students to be better prepared for the demands of higher level disciplinary and interdisciplinary learning.

\section{Interdisciplinary lessons for geography and beyond}

Through the collection of student perspectives and engagement on this interdisciplinary module, I have sought to develop an empirical study of how significant communicative competence is to interdisciplinary learning and, likewise, to identify certain domains in which students can develop such competence. In closing, it seems appropriate to comment on how successful the module was in developing communicative competence. Formal quantitative measures of learning gain collected by QMUL through final module grades and longitudinal student surveys show positive change in students' communicative competence. 95 per cent of students reported that their confidence in communicating complex ideas had improved because of the module, and 83 per cent reported that they had increased familiarity with concepts and approaches drawn from discipline other than their own. Exit grades for 75 percent of students on the module were higher than their benchmark scores drawn from previous modules. There was no significant difference in learning gain around communicative competence by 
disciplinary background. Capturing learning gain through such metrics is problematic, with exit grades providing little granular detail, and I treat conclusions drawn solely from these quantitative instruments with some scepticism.

Most students interviewed in the qualitative research also reported learning gains across several learning outcomes. The geographers in the group, overall, noted improvements across many of the domains and were more reflective in their learning than the students from other disciplines. While this is perhaps the result of the peculiar character of geography itself, with many undergraduate students immersed in differing disciplinary perspectives from both human and physical geography, it is perhaps also testament to the widespread promotion of communication skills and reflective learning by geography teaching staff (Haigh, 2001; Harrison, Short \& Roberts, 2003). Despite such positive results among the geography cohort, enabling learning across these key domains nonetheless requires the implementation of specific teaching and learning strategies. The "Health, Biomedicine, and Society" module discussed here has provided one empirical window onto interdisciplinary learning. In closing, however, I want to identify certain points for the consideration of those developing interdisciplinary classes and then raise three lessons for the discipline of geography based upon my experience of facilitating this module. As a disciplinary home for future learners, I argue, geography has an ongoing role in preparing geographers for interdisciplinary learning.

The 2015-2016 version of the module benefited from a broad variety of contributing disciplines, with significant student numbers from each discipline, and this created a positive atmosphere of interdisciplinarity. Future versions of the module with oscillating numbers of contributing disciplines and students may alter this dynamic and necessitate changes in the design and delivery of the module. In my own reflections on the module, I have identified two areas for refinement and improvement that others developing similar modules - both within and beyond geography - might want to consider incorporating into their own efforts. In future iterations of the module, I will give over more classroom time for reflective monodisciplinary group activities as preparation for interdisciplinary conversations later in the class. This would encourage learners to negotiate a "consensus" on how their discipline might approach a particular issue and, when discussed in conversation with other disciplines, would act to further emphasise the similarities and differences between disciplinary approaches. I hope to, for instance, task groups with creating introductory "guides" to their discipline that outline key perspectives, authors, and methodological approaches. Besides this monodisciplinary 
preparation, changes to the assessment structure will be a key development in future versions of the module. I feel that a formal group presentation would provide students with an opportunity to showcase collaborative research and their communicative competence. My experience in the 2015-2016 version of the module, however, suggests that the contribution of members in some group activities is uneven. As a result, part of the assessment in future will involve a group mark awarded to the collaborative research presentation, with an extra individual mark awarded to each student based on a written reflection on the successes and challenges of the collaborative research process.

In addition to refinements on this module, my experience of facilitating interdisciplinary learning has emphasised the duty of the home discipline to prepare learners for interdisciplinary study. With the rise of the interdisciplinary learning agenda occurring alongside the continued significance of the discipline as producer of knowledge and recruiter of students, home disciplines must be careful to support students who seek to learn across disciplines. First, and in preparing students for their experience of interdisciplinary learning, the discipline of geography has a key role in developing student awareness of disciplinary and interdisciplinary practices at an early stage. Students are introduced to common disciplinary perspectives and practices through core courses on the history and ideas of geography, but it is essential that module leaders frame these existing disciplinary narratives in relation to nascent interdisciplinary learning and research agendas. Such preparatory work might involve, for example, demonstrating how researchers borrow and adapt ideas by mapping the intellectual origin and evolution of key methods and concepts used in contemporary geographical research. Likewise, encouraging students to define key disciplinary concepts (such as "space," "place," and "justice") in small groups could be an informal method of developing disciplinary communication skills before they are needed in the production of assessed interdisciplinary outputs. Activities where groups of learners analyse exemplars of landmark texts or events in the discipline might also be useful to develop the disciplinary awareness that is an essential progenitor of communicative competence.

Second, the discipline of geography has a responsibility to provide learners with experience of interdisciplinary collaboration within and beyond the formal classroom setting. Whether individual departments frame this as an opportunity for undergraduate students to work together with peers from across the university to tackle a local issue or the chance to develop employability skills, further opportunities for interaction with those from outside the discipline 
is essential in challenging prevailing attitudes about other disciplines. Potential sources of this interdisciplinary experience could include: increasing joint supervision of undergraduate dissertation research by utilising the expertise of academics from different disciplines; extra institutional funding for student-led initiatives (such as conferences or film festivals) around pressing issues that bring together students from different disciplines; and, the promotion of action research to support local community research needs.

Third, and finally, the cultural and attitudinal changes that underpin much interdisciplinary learning evolve over long time periods, and these often exceed the duration of an individual interdisciplinary module. Lasting interdisciplinary learning thus requires forms of encouragement and support over an extended period, and home discipline staff can best provide these within disciplinary programme structures. How, then, can the discipline of geography support the continued development of interdisciplinary skills and abilities to which undergraduates have been introduced when a module ends? Students could, for example, score their familiarity on the seven domains of communicative competence following an interdisciplinary module to identify how their personal development might be further through modules and training available within geography. Systematic attention to these domains, alongside advice on how students might demonstrate progress in them, would promote a longer term engagement with interdisciplinary learning and reinforce the significance of communicative competence in the geography classroom and beyond.

\section{References}

Alptekin, C. (2002). Towards intercultural communicative competence in ELT. ELT Journal, $56,57-64$.

Baerwald, T. J. (2010). Presidential address: prospects for geography as an interdisciplinary discipline. Annals of the Association of American Geographers, 100, 493-501.

Barry, A., Born, G., \& Weszkalnys, G. (2008). Logics of interdisciplinarity. Economy and Society, 37, 20-49.

Becher, T., \& Trowler, P. R. (2001). Academic tribes and territories. Buckingham: Open University Press. 
Bracken, L. J., \& Oughton, E. A. (2006). “What do you mean?” The importance of language in developing interdisciplinary research. Transactions of the Institute of British Geographers, 31, 371-382.

Bradbeer, J. (1999). Barriers to interdisciplinarity: disciplinary discourses and student learning. Journal of Geography in Higher Education, 23, 381-396.

Byram, M. (1997). Teaching and assessing intercultural communicative competence. Clevedon: Multilingual Matters.

Callard, F., \& Fitzgerald, D. (2015). Rethinking interdisciplinarity across the social sciences and neurosciences. London: Palgrave Macmillan.

Canale, M. (1983). From communicative competence to communicative language pedagogy. In J. C. Richards \& R. W. Schmidt (Eds.), Language and communication (pp. 2-28). London: Routledge.

Castree, N. (2016). Geography and the new social contract for global change research. Transactions of the Institute of British Geographers, 41, 328-347.

Chomsky, N. (1965). Aspects of the theory of syntax. Cambridge: MIT Press.

Cook, I., et al. (2004). Follow the thing: papaya. Antipode, 36, 642-664.

Davies, M., Devlin, M., \& Tight, M. (Eds.). (2010). Interdisciplinary higher education: perspectives and practicalities. Bingley: Emerald.

Donovan, K., Sidaway, J. D., Stewart, I. (2011). Bridging the geo-divide: reflections on an interdisciplinary (ESRC/NERC) studentship. Transactions of the Institute of British Geographers, 36, 9-14.

Elder, G. (1999). "Queerying” boundaries in the geography classroom. Journal of Geography in Higher Education, 23, 86-93. 
Foster, J. (1999). What price interdisciplinarity? Crossing the curriculum in environmental higher education. Journal of Geography in Higher Education, 23, 358-366.

Haigh, M. J. (2001). Constructing Gaia: using journals to foster reflective learning. Journal of Geography in Higher Education, 25, 167-189.

Harrison, M., Short, C., \& Roberts, C. (2003). Reflecting on reflective learning: the case of geography, earth and environmental sciences. Journal of Geography in Higher Education, 27, 133-152.

Herrick, C. (2010). Challenging assumptions: teaching, documenting, producing and negotiating 'health'. Journal of Geography in Higher Education, 34, 345-362.

Hymes, D. (1972). On communicative competence. In J. Pride \& J. Holmes (Eds.), Sociolinguistics: selected readings (pp. 269-293). London: Penguin.

James, A. (2006). Critical moments in the production of "rigorous" and "relevant" cultural economic geographies. Progress in Human Geography, 30, 289-308.

James, R., \& McPhee, P. (2012). The whole-of-institution curriculum renewal undertaken by the University of Melbourne, 2005-2011. In P. Blackmore \& C. Kandiko (Eds.), Strategic curriculum change: global trends in universities (pp. 145-160). London: Routledge.

Jones, P. C., \& Merritt, J. Q. (1999). Critical thinking and interdisciplinarity in environmental higher education: the case for epistemological and values awareness. Journal of Geography in Higher Education, 23, 349-357.

Lau, L., \& Pasquini, M. (2008). "Jack of all trades"? The negotiation of interdisciplinarity within geography. Geoforum, 39, 552-560.

Lyall, C., Meagher, L., Bandola, J., Kettle, A. (2015) Interdisciplinary provision in higher education: current and future challenges. York: Higher Education Academy. 
Onsman, A., \& Newton, C. (2015). Blurred boundaries. Australian Universities' Review, 57, $37-45$.

Pharo, E., \& Bridle, K. (2012). Does interdisciplinarity exist behind the façade of traditional disciplines? A study of natural resource management teaching. Journal of Geography in Higher Education, 36, 65-80.

Pharo, E., Davison, A., Warr, K., Nursey-Bray, M., Beswick, K., Wapstra, E., \& Jones, C. (2012). Can teacher collaboration overcome barriers to interdisciplinary learning in a disciplinary university? A case study using climate change. Teaching in Higher Education, 17, 497-507.

Schmelzkopf, K. (2002). Interdisciplinarity, participatory learning and the geography of tourism. Journal of Geography in Higher Education, 26, 181-195.

Schoenberger, E. (2001). Interdisciplinarity and social power. Progress in Human Geography, $25,365-382$.

Scott, P. (2002). The future of general education in mass higher education systems. Higher Education Policy, 15, 61-75.

Simon, G. L., \& Graybill, J. K. (2010). Geography in interdisciplinarity: towards a third conversation. Geoforum, 41, 356-363.

Skole, D. L. (2004) Geography as a great intellectual melting pot and the preeminent interdisciplinary environmental discipline. Annals of the Association of American Geographers, 94, 739-743.

Strober, M. H. (2006). Habits of the mind: challenges for multidisciplinary engagement. Social Epistemology, 20, 315-331.

Woods, C. (2007). Researching and developing interdisciplinary teaching: towards a conceptual framework for classroom communication. Higher Education, 54, 853-866. 


\section{By the end of the module, you will be able to:}

Interdisciplinary abilities [after Woods (2007)]

- identify and apply appropriate methods and concepts from a variety of disciplines to examine an ethical issue in the study of health and biomedicine [conceptual competence];

- negotiate shared understandings of key terms and concepts from different disciplines [competence in negotiating meaning];

- negotiate different disciplinary oral and textual presentation styles and present collaborative research findings in a manner that is accessible to an interdisciplinary audience [competence in interdisciplinary text production].

Interdisciplinary cultures [after Byram (1997)]

- relate a document from another discipline to one from your own [interpreting and relating];

- acquire new knowledge of disciplinary cultures and practices outside of your own [attitudes];

- articulate the relevance of your own discipline, and those of others, to the study of health and biomedicine [discovery and interaction];

- critically evaluate the perspectives, practices and products of your own disciplinary culture and that of others [critical disciplinary awareness].

Table 1: "Health, Biomedicine, and Society" learning outcomes, adapted from Byram's (1997) and Woods' (2007) domains of communicative competence (in square brackets) 